\title{
TRAUMA MAMARIO POR MORDEDURA DE CABALLO - REPORTE DE CASO
}

\section{Breast injury due to horse bite - Case report}

Gloria Eugenia Camargo-Villalba, MD'; Pedro Manuel Bustamante-Bohigas, MD $^{2}$

Recibido: marzo 15/13 - Aceptado: noviembre 14/13

\section{RESUMEN}

Objetivo: presentar un caso de trauma mamario por mordedura de caballo en una mujer y revisar el manejo de este tipo de accidentes.

Materiales y métodos: se presenta un caso de trauma mamario izquierdo por mordedura de equino, de 10 días de evolución, en una mujer de 43 años de edad atendida en una institución de primer nivel de complejidad, posteriormente remitida para estudio imaginológico y manejo a una institución de tercer nivel de complejidad. Se realizó una búsqueda de la información en las bases de datos SciELO y PubMed con la terminología MeSH "Bite horsebreast trauma", y en español "trauma mamario por mordedura de equino".

Conclusiones: los traumatismos mamarios por mordedura de caballo son infrecuentes y pueden acompañarse de lesiones que van desde laceraciones, equimosis, hematomas, amputación de la glándula mamaria y complicaciones sistémicas de tipo infeccioso, así como secuelas severas de tipo funcional y estético. Es indispensable adoptar medidas preventivas generales y manejar secuencialmente el caso como se recomienda en el presente reporte.

1 Médica ginecoobstetra. Mastóloga. Docente, Facultad Ciencias de la Salud, Universidad de Boyacá, Colombia. Profesora titular. Tunja, Colombia.

2 Médico patólogo. Docente, Facultad Ciencias de la Salud, Universidad de Boyacá, Colombia. Profesor titular. Tunja, Colombia.
Palabras clave: mama, trauma, caballo, mordedura, hematoma, ultrasonido.

\section{ABSTRACT}

Objective: To present a case of female breast trauma due to horse bite and review the management of this type of injury.

Materials and methods: Case presentation of left beast injury caused by horse bite in a 43 year-old woman who came to a first level institution 10 days after the accident, and was then referred for imaging studies and management to a level 3 complexity institution. A search was conducted in the SciELO and PubMed databases using MeSH terms "Bite horse breast Trauma" in English and "mammary trauma due to equine bite" in Spanish.

Conclusions: Breast injury due to horse bite is infrequent and may be accompanied by lesions ranging from lacerations to bruising, hematomas, mammary gland amputation and infectious systemic complications, as well as severe functional and cosmetic sequelae. It is of the utmost importance to adopt general preventive measures and treat the case sequentially as recommended in this report.

Key words: Breast, trauma, horse, bite, hematoma, ultrasound.

\section{INTRODUCCIÓN}

La mama puede a menudo ser objeto de estímulos traumáticos, estos varían respecto a su naturaleza, 
intensidad y secuelas. La gran mayoría de ellos, por carecer de importancia clínica, no se describen en la literatura (1). En ocasiones, algunas lesiones se acompañan de importante morbilidad que puede poner en riesgo la vida del individuo afectado $(2,3)$.

En la actualidad no existe una clasificación de los traumatismos de mama universalmente aceptada; no obstante, en el trabajo de Uriburu et al., se presen$\tan 45$ casos de traumatismos de mama y el autor los clasifica en tres grupos: contusiones y heridas leves y moderadas; contusiones y heridas graves, y traumatismos por cuerpos extraños (4).

Las mordeduras ocasionadas por animales constituyen un tema de gran importancia clínica por la severidad de las secuelas y las complicaciones sistémicas que en ocasiones las acompañan, estas son principalmente producidas por animales domésticos como perros, gatos, hámster y caballos, y menos frecuentemente por animales no domésticos como serpientes, zorros, coyotes, murciélagos, simios (2). Las mordeduras de caballo pertenecen al grupo etiológico de origen mecánico y, por lo general, cuando se trata de seres humanos compromete los miembros superiores e inferiores, manos y cara $(5,6)$, y los afectados generalmente son personas que se relacionan con trabajos de cuidado y alimentación (7).

El presente caso clínico es importante porque el trauma mamario por mordedura de caballo constituye un evento que requiere un manejo multidisci-
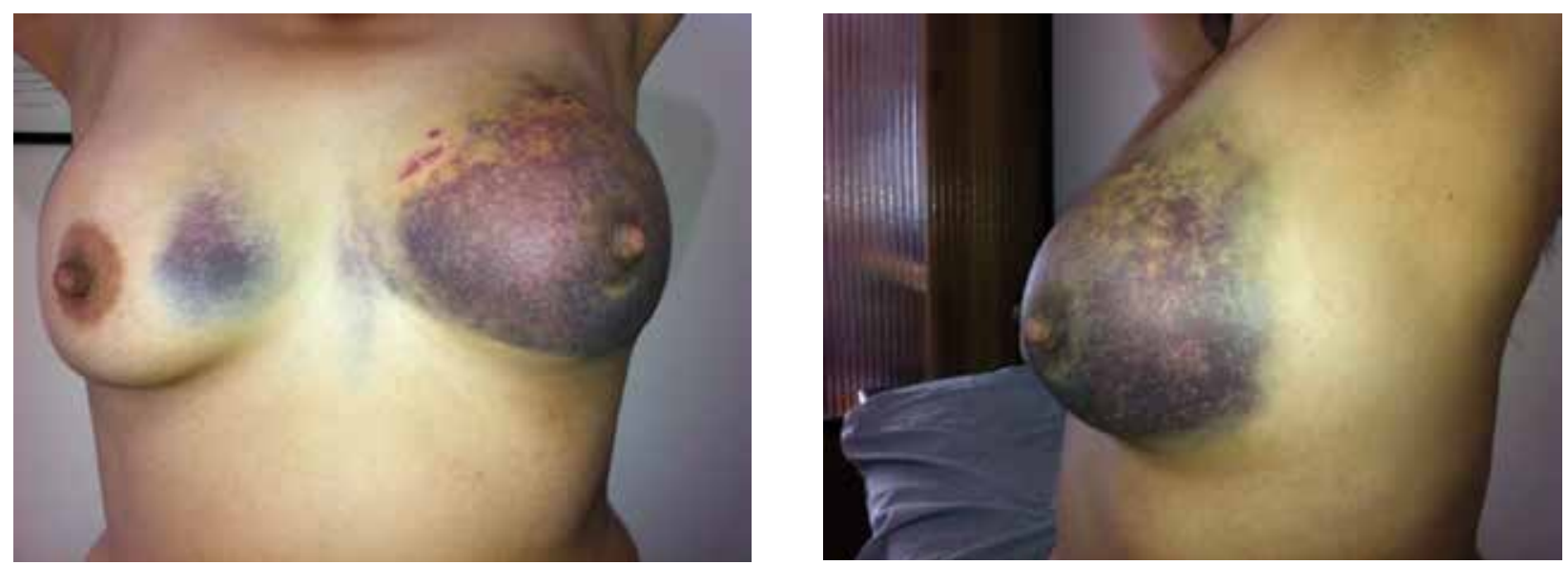

plinario. El tipo de trauma es un evento infrecuente reportado en la literatura (3) y es indispensable que el médico esté. El objetivo de la presentación de este caso es revisar el manejo de este tipo de accidentes.

\section{CASO CLÍNICO}

Paciente femenina procedente de área rural, de 43 años, quien consulta por trauma de 10 días de evolución consistente en mordedura accidental por su propio caballo en la mama izquierda, produciendo dolor generalizado de intensidad 10/10 en la mama que se propaga a la región axilar ipsilateral y gran equimosis de la misma.

$\mathrm{Al}$ examen físico se encuentra paciente álgida, con signos vitales en parámetros normales, observándose equimosis que compromete el 100\% de la mama izquierda y otra equimosis en horas 2 a 5 de la mama derecha. En el cuadrante supero interno de la mama izquierda presenta tres lesiones dérmicas, dos de forma lineal y una de forma circular con costras, las lesiones se encuentran próximas entre sí; las lineales tienen $17 \mathrm{~mm}$ de longitud y la circular 7 mm de diámetro (figura 1). A la palpación de la mama izquierda se detecta masa dolorosa de 10 centímetros de diámetro que compromete el 70\% del volumen mamario. La palpación de la mama derecha es dolorosa en los cuadrantes internos, no se detectan masas. Las regiones axilares no presentan hallazgos patológicos.

Figura 1. Frente y perfil de trauma mamario 
Se efectúa estudio de ultrasonido con transductor lineal de $10 \mathrm{MHz}$ observándose un nódulo hipoecoico, de bordes anteriores ligeramente lobulados, en su mayor parte netos, que mide $3,35 \mathrm{~cm}$ de diámetro transverso mayor, sugestivo imaginológicamente de un hematoma (figura 2).

Se inicia manejo con antibioticoterapia (cefalexina $500 \mathrm{mg}$ cada 6 horas), toxoide antitetánico y se deriva para el respectivo drenaje del hematoma. La paciente asiste nuevamente a control después de un año de sucedido el evento, clínicamente asintomática; se le realiza estudio ultrasónico, encontrándose el mismo en límites normales.

\section{MATERIALES Y MÉTODOS}

Con base en la siguiente pregunta: ¿Cuál es el manejo del trauma mamario por mordedura de caballo?, se realizó una búsqueda de la información en la base de datos Medline / vía PubMed y SciELO, con los términos "horse bite, breast trauma, and management or treatment". Se analizaron: reporte de casos, capítulos de libro, guías de manejo y artículos de revisión de los años comprendidos de 1968 a 2013.

Aspectos éticos. Se contó con autorización de la paciente para la publicación del caso y se garantizó la confidencialidad de la información.

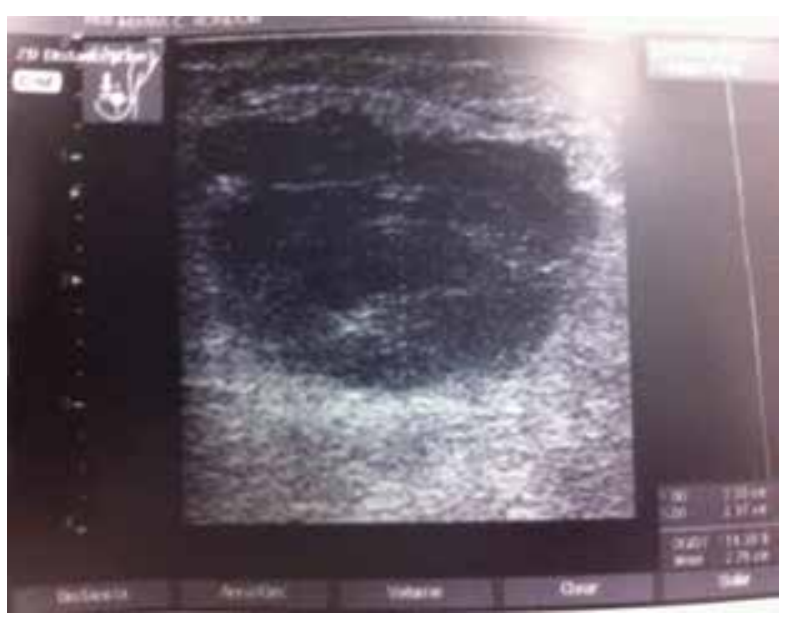

Figura 2. Ecografía mamaria

\section{RESULTADOS}

Se encontraron ocho artículos, tres guías de manejo, un caso clínico y un capítulo de libro.

\section{DISCUSIÓN}

El presente caso es el primero publicado en Colombia de trauma mamario de origen mecánico por mordedura de caballo con laceraciones cutáneas y extenso hematoma sin ablaciones, sin reacciones agudas inmunológicas ni alteraciones sistémicas de tipo infeccioso. Este caso corresponde al grupo denominado "contusiones y heridas graves" (4). De interés es el hecho de que la víctima tiene una relación cercana con el animal. El área comprometida por el trauma no es el sitio de mayor frecuencia reportado en la literatura (5).

Estas heridas pueden ser superficiales o profundas y, en ocasiones, acompañarse de equimosis, hematomas e incluso extensas pérdidas tisulares (8). De especial interés es la posibilidad de que las heridas por mordedura de caballo pueden ser el mecanismo de transmisión de microorganismos patógenos como S. aureus, Neisseria sp, Pasteurella caballii, Streptococcushyicus, Streptococcusanginosus, Actinobacilluslignieressii, B. fragilis, Bacteroide sureolytica, E. coli, Prevotella melanogenicus y Prevotella heparinolytica (2). Una complicación infrecuente pero de gran importancia médica es que estas heridas pueden acompañarse de una reacción anafiláctica desencadenada por el efecto antigénico de las lipocalinas, proteínas que se encuentran en grandes concentraciones en la saliva del caballo (9).

Se encontró un caso en la literatura (8) en el que la mordedura del caballo se acompañó de amputación, lo que hizo necesario tratamiento quirúrgico reconstructivo. Los autores consideran que esta situación se presenta probablemente debido a la diferencia de volumen de las mamas; sin embargo, esta hipótesis no se puede confirmar por cuanto la publicación del doctor Ho Quoc no describe en detalle el volumen de la mama amputada. 
Imágenes diagnósticas. Un aspecto para tener en cuenta es el análisis comparativo entre el examen físico y el hematoma observado en el ultrasonido, situación que se explica por el edema de tejido celular subcutáneo asociado al trauma adyacente al hematoma. A pesar de presentar una lesión que compromete la barrera natural dérmica, y que la saliva de los caballos puede transportar múltiples microorganismos patógenos (2), en este caso no se presentó proceso infeccioso local ni sistémico.

Es importante insistir en la necesidad de un interrogatorio adecuado con el fin de obtener información sobre el contexto epidemiológico donde se presenta el evento adverso para poder orientar un adecuado manejo terapéutico; de igual forma, es indispensable efectuar los estudios imaginológicos ante situaciones similares con el propósito de establecer un diagnóstico y manejo adecuados.

Ante casos similares los autores recomiendan efectuar estudios imaginológicos pre y postratamiento con el propósito de establecer la evolución de las lesiones que permitan identificar lesiones características por mordedura de caballo

En cuanto a la prevención del trauma por mordedura de caballo se recomienda a las personas que establecen un contacto cercano con estos animales considerar el uso de cascos protectores o sombreros de ala ancha, guantes, chalecos y ropa de jean (10). Respecto al manejo se debe llevar a cabo una exhaustiva limpieza con solución salina y debridación si la herida lo amerita; ante signos de infección local se deben tomar muestras para estudios microbiológicos e instaurar una terapia antibiótica con amoxicilina y ácido clavulónico, así como profilaxis antitetánica, si la herida lo amerita debe establecerse interconsulta con cirugía (11).

\section{CONCLUSIONES}

Los traumatismos mamarios por mordedura de caballo son infrecuentes y pueden acompañarse de lesiones que van desde laceraciones, equimosis, hematomas, amputación de la glándula mamaria y complicaciones sistémicas de tipo infeccioso y alérgico, así como de secuelas severas de tipo funcional y estético.

Es indispensable adoptar medidas generales de prevención cuando se establece cercanía con los caballos.

El manejo recomendado está orientado a efectuar estudios microbiológicos de acuerdo con el tipo de lesión, medidas antisépticas, profilaxis antitetánica, antibioticoterapia y manejo quirúrgico, según el caso.

\section{REFERENCIAS}

1. Sierra A, Piñero A, Illana J. Guías Clínicas de la Asociación Española de Cirujanos. Traumatismos de la Mama. 10 edición. España: Aran Ediciones; 2006.

2. Jofre LM, Parret C, Abarca K, Solari V, Olivares R, López J. Recomendaciones para el manejo de mordeduras ocasionadas por animales. Comité de infecciones emergentes Sociedad Chilena de Infectología. Rev Chi Infect. 2006;23:20-34.

3. Vidal S, Barcala L, Tovar JA. Horse byte injury. Eur J Dermatol. 1998;8:437-8.

4. Uriburu JV, Gómez M, Macagno A, Margossian J, Rojas RR, Uriburu JL, et al. Traumatismos de la mama. Rev Argent Cir. 1994;66:140-5.

5. Fernández S, Rodríguez I, Núñez M, Moreno R, Ledo A. Celulitis por Pasteurella multocida Actas DermoSifiliográficas 1977;88:31-4.

6. Weber DJ, Wolfson JS, Swartz MN, Hooper DC. Pasterella multocida Infections. Report of 34 cases and review of the literature. Medicine (Baltimore). 1984:63:133-54.

7. Muñiz M, GonzálezJD, Veiga A, RodríguezA. Accidentes graves provocados por caballos. Alertas y pautas de prevención. Anales de Pediatría 2009;70:434-7.

8. Quoc CH, Meruta A, La Marca S, Fabiano L, Toussoun G, Delay E. Breast amputation correction of a horse bite using the lipomodeling technique. Aesthet Surg J. 2013;33:93-6. 
9. Guida G, Nebiolo F, Helfer F, Bergia R, Rolla G. Anaphylaxis after a horse bite. Allergy. 2005 60:1088-9.

10. Emet M, Beyhun NE, Kosan Z, Aslan S, Uzkeser M, Cakir ZG. Animal-related injuries: epidemiological and meteorological features. Ann Agrir Environ Med. 2009;16:87-92.
11. Köse R, Sögut Ö, Mordeniz C. Management of horse and donkey bite wounds: a series of 24 cases. Plast Reconstr Surg. 2010;125:251e-2e.

\section{Conflicto de intereses: ninguno declarado.}

